\title{
Molecular imaging: the emerging role of optical imaging in nuclear medicine
}

\author{
Fijs W. B. van Leeuwen - Marion de Jong • on behalf of the EANM Translational \\ Molecular Imaging Committee; Marion de Jong, Fijs van Leeuwen, Tony Lahoutte, \\ Laura Evangelista, Jacques Barbet, Silvana Del Vecchio, Roger Schibli.
}

Published online: 19 July 2014

(C) Springer-Verlag Berlin Heidelberg 2014

Through its role in (routine) patient care, nuclear medicine has set the standard for molecular imaging: the sensitivity and specificity of the available (multimodality) nuclear imaging technologies (SPECT/CT, PET/CT and PET/MR) are superior to those of any other clinical imaging modality. Nevertheless, efforts are ongoing to apply ultrasound, magnetic resonance and/or optical imaging techniques to molecular imaging in the clinic. A careful look at the theoretical detection sensitivities of the individual modalities readily confirms that especially ultrasound, optical and radionuclide techniques currently have the potential to visualize (low) biomarker expression levels using imaging probes [1]. Having said this, there are also activities regarding the development of MR contrast agents. Since the value of ultrasound technologies remains confined to the vascular network and the sensitivity of MR is still relatively low, only optical imaging can, in theory, match the sensitivity performance of nuclear medicine techniques.

\section{Why optical imaging?}

Although these claims are not always directly in line with our own opinion, various authors have suggested during the recent revival of optical imaging that optical technologies can replace techniques currently used in clinical routine for some in vivo applications [2]. A number of arguments have been put

\footnotetext{
F. W. B. van Leeuwen $(\bowtie)$

Interventional Molecular Imaging Laboratory, Department of

Radiology, Leiden University Medical Center, Leiden,

The Netherlands

e-mail: f.w.b.van_leeuwen@lumc.nl

M. de Jong

Department of Nuclear Medicine/Radiology, Erasmus MC,

Rotterdam, The Netherlands
}

forward to substantiate the development of optical imaging technologies for in vivo use:

- Reduced side effects: Since optical tracers generally do not use radioactivity, the potential for side effects is limited.

- Limited facility requirements: Production of optical tracers does not require dedicated radiochemistry laboratories or, in the case of PET tracers, cyclotron facilities.

- Lower financial cost: The costs of surgical fluorescence cameras are, on average, around 100,000 euros, is markedly lower than those of preclinical SPECT/CT, PET/CT or PET/MRI cameras.

- The ability to directly translate fluorescence technologies available for molecular cell biology applications to the in vivo situation: A great number of fluorescent tracers (e.g. $\mathrm{mAbs}$ ) are available for the microscopic visualization of biomarkers in vitro and at histology.

- The availability of optical reporter markers to monitor disease spread and progression: Via genetic transcription, tumour cells or even whole animals can be genetically modified so that they express a reporter gene. Although reporter gene imaging is also possible in combination with PET or SPECT, to date use of the optical read-out mechanism is more widespread. Optical read-out mechanisms are bioluminescence enzymes such as luciferase, which, via a reaction with the injected substrate luciferin, produce light, or one of the fluorescent proteins e.g. green fluorescent protein (GFP). The latter require light excitation to generate a light emission (see below for more detail on bioluminescence and fluorescence).

- The potential to optically identify diseased areas during interventional procedures: Light-sensitive fluorescence cameras provide video-rate feedback, enabling real-time visualization of superficially located areas of disease. 
- The potential to increase the tissue penetration of optical emissions by using near-infrared emissions: The signal penetration of emissions between 400 and $650 \mathrm{~nm}$ is confined to the millimetre range, while near-infrared emissions may penetrate tissue as far as $1 \mathrm{~cm}$.

- The potential for superior in vivo resolution [1]: When used superficially, fluorescence resolution can be less than $1 \mu \mathrm{m}$ while radionuclide-based techniques remain limited to the millimetre-centimetre range. This feature can be used to depict the subcellular localization in early analysis of, for example, intraluminal lesions of the colon. It also offers the potential to histologically identify microscopic disease, e.g. at surgical margins.

\section{Limitations of optical imaging}

Despite the "promise" of optical technologies, clinical studies in the field of image-guided surgery have demonstrated that purely optical identification of, for example, surgical margins is only of value when the features investigated lie within a known anatomy, as tissue penetration of fluorescent signals is limited to the centimetre range [3]. When attempts are made to identify signals with an unknown (or deep) origin, or when the surgical margins need to be determined below the resection surface, the limited tissue penetration limits application of optical technology in a minimally invasive approach. In such cases, guidance from preoperative modalities is required. Signal attenuation also severely limits the quantification possibilities for optical signals. For reference, PET is an autoradiographic 3-D methodology which provides an in-depth total body view and allows absolute signal quantification in vivo.

\section{Optical imaging as an add-on to nuclear imaging}

To obtain additional information, optical imaging can be used in parallel or in combination with nuclear imaging (Fig. 1). One of the most straightforward and perhaps most widespread multimodal applications of optical approaches in preclinical nuclear medicine research is the use of disease models using tumour cell lines genetically transfected with, for example, fluorescence proteins and/or bioluminescence enzymes [4, 5]. In our experience, genetically generated optical signals can be use as sensitive reference signals to longitudinally monitor disease onset/spread in animal models. Bioluminescence is valuable for optical screening purposes since it is not obscured by autofluorescence and only occurs in transfected cells. It relies on the intracellular conversion of, for example, luciferin, rather than light excitation, to generate an optical signal. Since genetic modification techniques cannot easily be translated to the clinic, dedicated tracers with an optical signature are required to make optical imaging also possible in patients. In our view, it is most logical to initially derive such optical tracers from proven radiotracers. Since Cerenkov imaging uses light emission caused by $\beta$-particles travelling through a medium, detection of these signals seems to be the most straightforward technique to include an optical imaging readout in a nuclear medicine setting. This technique can be applied to known PET tracers such as ${ }^{18}$ F-FDG [6]. While the low photon flux (amount of emitted light) and the unfavourable wavelength (the peak of the emitted light lies in the wavelength area with maximal tissue absorbance, $<400 \mathrm{~nm}$ ) are major limiting factors, the technique has nevertheless already been successfully applied in both preclinical and clinical settings.

Alternatively, hybrid (combined radioactive and fluorescent) derivatives of known radiotracers can be developed. Because fluorescent dyes are incorporated in these designs, the photon flux is about three orders of magnitude larger than Cerenkov emissions, and the wavelength of the emission can be tuned by altering the structure of the organic dye [3]. Given the size and lipophilicity of the most common fluorescent dyes, it is likely that the introduction of fluorescent groups will lead to probe affinities and distribution profiles that differ from the parent radiotracer. One may reason that instead of hybrid probes, probe pairs could be applied for sequential preoperative nuclear imaging and intraoperative fluorescence imaging. However, a practical limitation of fluorescent probes is the restricted ability to quantify their kinetics and biodistribution profiles during the preclinical evaluation process. Fluorescence cannot be used for true quantitative pharmacokinetic and distribution studies. We found that the radioactive component of hybrid tracers can compensate for this shortcoming, either as an intermediate step or as final product [7]. The quantitative feedback provided by hybrid tracers can therefore help optimize the kinetics, biodistribution and dose in relation to signal intensity. Note: a direct correlation between the doses needed for nuclear medicine-based imaging and the signal intensities obtained with fluorescence will help in assessing whether fluorescence tracers can be clinically applied at a tracer dose.

\section{Tracer production and translation}

The availability of dedicated tracers has driven the successes achieved in nuclear medicine and the same will likely be true for the future of fluorescence imaging. During recent decades, research efforts have yielded numerous preclinically evaluated radiotracers for molecular imaging approaches focusing on oncology, cardiology, neurology, infections, etc. Successful translation of these efforts has resulted in a significant number of first-in-human trials and in some cases also reimbursement. This nuclear medicine history has provided vast expertise in 



Fig. 1 Schematic representation of the different types of optical emission in relation to radioactive emissions. a Radioactive emission of a gamma or a beta ${ }^{+}$coming from a radionuclide. b Enzymatic conversion of a substrate leading to the emission of bioluminescence light. $\mathbf{c}$ Excitation of

radiochemistry, GMP production, structure-activity relationships, etc. Since organic chemistry provides the basis for the development of both radiotracers and fluorescent tracers suitable for human, the chemical challenges are similar in both approaches; maintaining the functionality and favorable kinetics of the targeting moiety is vital when introducing the imaging label. We are therefore of the opinion that expertise in radiochemistry may aid the development and subsequent clinical translation of new types of molecular imaging tracers, such as optical tracers, optical/nuclear hybrid tracers and MR contrast agents.

\section{Future outlook}

In our opinion, the future of fluorescence imaging is radiant. Hybrid derivatives of known radiotracers and/or Cerenkovbased detection of radiotracers can be expected to be used in the field of image-guided surgery [8]. At the same time, optical imaging may help add detail during the initial evaluation of new disease-specific tracers. However, significant chemical efforts are required to make the affinities/ a fluorescent dye resulting in the emission of light. d Generation of Cerenkov emission by beta-particles travelling through a medium. e Combining a radioactive and a fluorescent group on one molecule yields radiotracers with an optical signature

distribution patterns of fluorescent and hybrid tracers competitive with those of known radiotracers. When the detection technology for Cerenkov imaging is improved with respect to sensitivity, specificity and speed, an almost unlimited optical imaging opportunity may emerge.

\section{Concluding remarks}

In clinical routine the diagnosis of patients is rarely based on the use of a single imaging modality - multimodality imaging can provide additional information on different features, on morphological and functional as well as molecular characteristics. Since optical imaging offers some unique possibilities, we expect this technology to be of complementary value in the field of nuclear medicine. Clear examples are: (1) availability of a genetically encoded reference for disease spread in preclinical models, (2) ability to analyse tracer localization and kinetics at the cellular and subcellular levels, and (3) real-time optical guidance during surgery.

Acknowledgments This editorial is endorsed by the European Radio guided Surgery Network (http://www.radioguidedsurgery.net). 


\section{References}

1. Meikle SR, Kench P, Kassiou M, Banati RB. Small animal SPECT and its place in the matrix of molecular imaging technologies. Phys Med Biol. 2005;50:45-61.

2. Crane LM, Themelis G, Arts HJ, Buddingh KT, Brouwers AH, Ntziachristos V, et al. Intraoperative near-infrared fluorescence imaging for sentinel lymph node detection in vulvar cancer: first clinical results. Gynecol Oncol. 2011;120:291-5.

3. Chin PT, Welling MM, Meskers SC, Valdes Olmos RA, Tanke H, van Leeuwen FW. Optical imaging as an expansion of nuclear medicine: Cerenkov-based luminescence vs fluorescence-based luminescence. Eur J Nucl Med Mol Imaging. 2013;40:1283-91.
4. Weissleder R, Pittet MJ. Imaging in the era of molecular oncology. Nature. 2008;452:580-9.

5. Negrin RS, Contag CH. In vivo imaging using bioluminescence: a tool for probing graft-versus-host disease. Nat Rev Immunol. 2006;6: 484-90.

6. Thorek DL, Riedl CC, Grimm J. Clinical Cerenkov luminescence imaging of (18)F-FDG. J Nucl Med. 2014;55:95-8.

7. Kuil J, Buckle T, van Leeuwen FW. Imaging agents for the chemokine receptor 4 (CXCR4). Chem Soc Rev. 2012;41: 5239-61.

8. Brouwer OR, van den Berg NS, Mathéron HM, van der Poel HG, van Rhijn BW, Bex A, et al. A hybrid radioactive and fluorescent tracer for sentinel node biopsy in penile carcinoma as a potential replacement for blue dye. Eur Urol. 2014;65:600-9. 